\title{
Leserbrief zum Beitrag: Kappstein I. Mund-Nasen-Schutz in der Öffentlichkeit: Keine Hinweise für eine Wirksamkeit Krankenhaushygiene up2date 2020; 15: 279-297
}

Sehr geehrte Frau Kappstein,

mit Interesse habe ich Ihre Übersichtsarbeit „Mund-Nasen-Schutz in der Öffentlichkeit: Keine Hinweise für eine Wirksamkeit“ im aktuellen Heft 3 von „Krankenhaushygiene up2date“ vom September 2020 [1] gelesen. Ich kenne Ihre begründeten Auseinandersetzungen mit Empfehlungen der Kommission für Krankenhaushygiene und Infektionsprävention (KRINKO) am Robert Koch-Institut [2], schätze Ihren Sachverstand [3] und Ihr kritisches Hinterfragen von Empfehlungen. Auch unter Berücksichtigung des mangelnden expliziten Wissens zu SARS-CoV-2 im Frühjahr dieses Jahres und des schnellen Zuwachses im Laufe des vergangenen halben Jahres bin ich über einige Ihrer Statements irritiert und mit Ihren Schlussfolgerungen nicht einverstanden und will begründen, warum.

\section{Diskrepanz zwischen analytischer Sorgfalt und unausgewogenen bzw. partiell apodiktischen Schlussfolgerungen}

Ich teile Ihre Aussagen zur HongkongStudie [4], die an Patienten mit respiratorischen Symptomen unterschiedlicher Infektionen (durch Influenzaviren, Rhinoviren und saisonale humane Coronaviren) durchgeführt wurde und deren fraglicher Übertragbarkeit auf die aktuelle SARS-CoV-2-Pandemie. Ihre Erörterungen der WHO-Empfehlungen von 2019 zum Schutz der Allgemeinbevölkerung bei schweren Epi- und Pandemien [5], der WHO-Empfehlung zu Covid-19 $[6,7]$ und der ECDC-Empfehlungen [8] sind korrekt, auf die in Teilen unterschiedlichen Empfehlungen der CDC [9] gehen Sie jedoch nicht differenzierter ein.

Sie zitieren aus den RKI-Publikation vom 14.4.20 „Mund-Nasen-Bedeckung im öffentlichen Raum als weitere Komponen- te zur Reduktion der Übertragungen von COVID-19“ [10]: „Eine teilweise Reduktion dieser unbemerkten Übertragung von infektiösen Tröpfchen durch das Tragen von MNB könnte auf Populationsebene zu einer weiteren Verlangsamung der Ausbreitung beitragen “ [10] und kritisieren die „könnte“-Formulierung als „eine Formulierung, die im wissenschaftlichen Diskurs wegen offensichtlich fehlender Belege für eine Empfehlung mit weitreichenden Folgen eigentlich nicht hätte verwendet werden dürfen“ [1] (Seite 285). Letzteren Satzkann man so nicht stehen lassen. Im wissenschaftlichen Diskurs werden bei Empfehlungen in Leitlinien sowohl Evidenz als auch Grad oder Stärke der Empfehlung (GRADE-Klassifikation [11] angegeben, kann-Empfehlungen sind durchaus erlaubt.

Die RKI-Empfehlung „Mund-Nasen-Bedeckung im öffentlichen Raum als weitere Komponente zur Reduktion der Übertragungen von COVID-19“ mit dem Untertitel „Strategie-Ergänzung zu empfohlenen Infektionsschutzmaßnahmen und Zielen (3. Update)“ [9] ist im Stil einer kurzen Mitteilung (2,5 Seiten) an interessierte Laien und die breite Öffentlichkeit konzipiert, enthält 9 Literaturzitate und stellt keine mit GRADE-Klassifikation [11] versehene wissenschaftliche Leitlinie dar. Die WHO nennt ihre Veröffentlichungen vom 6. April und 5. Juni 2020 $[6,7]$ „interim guidance“ und ihr Update vom 9.7.20 zu „Transmission of SARSCoV-2: implications for infection prevention precautions“ einen „scientific brief“ [12]. Auch wenn Titel und Darstellung der RKI-Mitteilung vom 14.4.20 [10] auf die MNB klar als „weitere Komponente zur Reduktion der Übertragungen von COVID-19“ hinweisen, ist Ihre Anmerkung richtig: „transparent wäre es gewesen, auf das Fehlen von wissenschaftlichen Daten für den generellen Einsatz von MNB im öffentlichen Raum ausdrücklich hinzuweisen. Wenigstens aber hätte im letzten Satzdes Artikels, wie überall zuvor im Text, nur davon gespro- chen werden sollen, dass der MNB ein Baustein sein könnte, um Übertragungen zu reduzieren, nicht aber dies als Tatsache zu formulieren “ [1] (Seite 288).

So löblich es ist, über die Empfehlungen des BfArM zum Umgang mit Masken [13] hinaus ausführlich auf Nachteile und den korrekten Umgang mit Masken hinzuweisen, für die Vielzahl der von Ihnen aufgeführten möglichen Nachteile von Masken führen Sie jedoch keine einzige Studie an, die den Nachweis einer Erhöhung der SARS-CoV-2-Übertragung durch Kontaktinfektionen belegt - sei es direkt über Selbstinokulation von virushaltigem Material auf Nasenschleimhaut oder Bindehaut des Auges, sei es indirekt über Kontamination von Flächen, von denen sich andere Personen via Handkontakt und nachfolgendem Gesichtskontakt infizieren. Eine Übertragung via Kontakt mit kontaminierten Flächen wird wegen mangelnder Übereinstimmung experimenteller Daten mit Alltagssituationen als überschätztes Risiko angesehen [14]. Inwieweit ihre infektiologischen Bedenken bezüglich einer theoretisch möglichen Infektionsübertragung bei falschem Gebrauch von Masken de facto eine Rolle spielen, ist durch keine Beobachtungsstudie und keinen anekdotischen Hinweis belegt, ist also völlig offen und ungeklärt. Ihr unter der Überschrift „Schlussfolgerungen für die Anwendung von Masken im öffentlichen Raum“ als Merksatz markiertes Statement „Der Gebrauch von Masken im öffentlichen Raum ist schon allein aufgrund des Fehlens von wissenschaftlichen Daten fragwürdig. Zieht man dazu noch die erforderlichen Vorsichtsmaßnamen in Betracht, müssen (Unterstreichung $\mathrm{OL}$ ) nach den aus Krankenhäusern bekannten Regeln im öffentlichen Raum sogar als ein Infektionsrisiko betrachtet werden“ [1] (Seite: 292). 2 Sätze später dann: „Denn ganz gleich, ob Pflicht für alle Bürger oder freiwillig getragen von allen Bürgern, die das aus welchen Gründen auch immer wollen, 
bleibt es ein Faktum (Unterstreichung $\mathrm{OL})$, dass Masken in der Öffentlichkeit mehr Schaden als Nutzen bringen können “ [1] (Seite 292). Ein Faktum? Eine Tatsache? Ohne Belege ist dies kein Faktum, sondern eine Behauptung, die im Widerspruch zu Empfehlungen internationaler Experten steht [15-17]. Ich kann Sie nur mit Ihrer oben zitierten eigenen Aussage konfrontieren: „Transparent wäre es gewesen, auf das Fehlen von wissenschaftlichen Daten ...(hier: dass Masken im öffentlichen Raum als Infektionsrisiko betrachtet werden müssen und dass Masken in der Öffentlichkeit mehr Schaden als Nutzen bringen können [Einfügung $\mathrm{OL}$ ]) ... ausdrücklich hinzuweisen. Wenigstens aber hätte ... nur davon gesprochen werden sollen, dass ... sein könnte, ... nicht aber dies als Tatsache zu formulieren" [1] (Seite 288). Hier hätte ich mir mehr Ausgewogenheit und Sorgfalt in der Wortwahl gewünscht.

Auch wenn Ihre Anfang Juli entstandene Übersichtsarbeit den damaligen Wissenstand reflektiert, einige Monate später sieht manches (z.B. Aerosolübertragung) etwas anders aus, was mit kleinen Korrekturen bei Sichtung der Druckfahnen oder einem Addendum-Hinweis hätte relativiert werden können.

\section{Wissenschaftstheoretische Aspekte: Wie Wissenschaft- ler Krankheiten erklären}

Ihr Fokus auf evidenzbasierte Fakten wird in meinen Augen der komplexen Situation des Auftretens einer neuen Infektionskrankheit, die sich von einer Epidemie zu einer Pandemie ausgeweitet hat, der Klärung ihrer Pathogenese und der Erarbeitung von wirksamen Präventionsund Therapiemaßnahmen nicht gerecht. Wie der Kognitionswissenschaftler und Philosoph Paul Thagard in seinem Buch „How scientists explain disease“ [18] am Beispiel der (Wieder-) Entdeckung von Helicobacter pylori und der Klärung der Ulkusgenese erläutert, greifen bei der Abklärung einer Krankheit verschiedene Faktoren wie klinische Beobachtung, pathophysiologische Überlegungen, experimentelle Ergebnisse, widerstreitende Hypothesen u.a.m. ineinander und ver- stärken sich gegenseitig. Wissenschaft ist ein komplexes Zusammenspiel vieler Subdisziplinen mit ihren speziellen Methodiken, ein mäanderndes Zusammenfügen aus unterschiedlich großen und unterschiedlich wichtigen Befunden zu einer plausiblen Hypothese, ein Testen und Überprüfen von Modellen hin zu einem kohärenten Gesamtbild [18-20]. Zu den mentalen Taktiken einer wissenschaftlichen Herangehensweise zählt G. N. Derry das Modellieren von Modellen hin zu sukzessiver Approximation und die gedankliche Stringenz bei der Klärung von Evidenz, Gründen und kritischer Evaluation [20].

In wissenschaftstheoretischer Sicht ist in meinen Augen Ihre Abwertung von theoretischen Modellierungsstudien nicht berechtigt. Modellierungsstudien sind für Epidemiologen und Public Health-Experten notwendige Hilfen bei schwierigen gesundheitspolitischen Entscheidungen zur Pandemieeindämmung. Aus dem Vergleich von Modellrechnungen, die ein Bündel an präventiven Maßnahmen berücksichtigen [15,21-22], mit anderen Modellrechnungen, die lediglich eine Maßnahme wie Abstandhalten [2325], z.T. unter Einbeziehung situationsund altersabhängiger Kontakte [23], differenziert analysieren, lassen sich durchaus Rückschlüsse auf eine mögliche Rolle dieser Maßnahme hinsichtlich der Ausbreitung der Infektion ziehen. In Modellrechnungen aus 15 unterschiedlichen amerikanischen Staaten und Washington, in denen ein Tragen von Masken im öffentlichen Raum (z.B. Innenräumen von Einzelhandelsgeschäften, Ein- u. Durchgangsbereich in Restaurants, AuBenräumen wie Wartezonen vor Geschäften, Bus- und S-Bahnhaltestellen, geschlossenen Räumen wie öffentlichen Verkehrsmitteln, Taxis u. a.m.) angeordnet wurde, wurde für den untersuchten Zeitraum (8. April und 15. Mai 2020) ein die Infektionsausbreitung verlangsamender Effekt gegenüber der Vorperiode beschrieben. Eine Hochrechnung ergab, dass durch die Maskenpflicht in den betreffenden Staaten der USA vermutlich 230000 - 450000 Covid-19-Fälle hätten vermieden werden können [26]. Physikalisch-aerodynamische Untersuchungen und experimentelle Untersuchungen be- legen die Wirksamkeit von Masken gegen Infektionsübertragungen durch Tröpfchen und Aerosole [27-30]. Einzelbeobachtungen unter Alltagsbedingungen zeigen die Effektivität von Masken in der Verhinderung einer SARS-CoV-2Übertragung [31].

Beim derzeitigen Kenntnisstand zu Covid-19 benötigen wir zur Lösung des Puzzles und zur vollständigeren Klärung des Krankheitsbilds alle Arten von Bausteinen einer Evidenz, nicht nur die in der Evidenz-Hierarchie weit obenstehenden Bausteine [32]. Wissend, dass „absence of evidence is not evidence of absence“ plädiere ich statt engem Fokus auf randomisierte klinische Studien für eine breitere Sicht mit Einbeziehung aus Ergebnissen anderer Fachdisziplinen und für eine intensive interdisziplinäre Zusammenarbeit.

\section{Infektiologisch-patho-} physiologische Aspekte: Dosis-Wirkungsbeziehungen und die „Variolation“-

\section{Hypothese}

Sie weisen mit Recht darauf hin, dass mit SARS-CoV-2-infizierte Personen bereits vor Auftreten von Symptomen (potenziell) infektiös sind und dass dies von anderen Viruserkrankungen wie z. B. Influenza und Masern schon lange bekannt ist. Infektiologisch schon lange bekannt sind auch tierexperimentelle Befunde zu Dosis-Wirkungs-Beziehungen zwischen Anzahl infizierender Viren und Krankheits- bzw. Mortalitätsverlauf. Das Konzept der letalen Dosis 50, d.h. der Virusdosis, bei der $50 \%$ der infizierten Tiere sterben, gilt auch für Coronaviren, die das Middle East Respiratory Syndrome (MERS) verursachen [33]. Im Rahmen einer Impfstoffentwicklung haben gesunde Freiwillige, die unterschiedlichen Dosen eines Wildtyp Influenza A-Virus ausgesetzt waren, stärkere Symptome bei höheren Virus-Inocula entwickelt [34].

Derzeit ist unklar, warum zu Beginn der Pandemie im April die Anzahl asymptomatisch mit SARS-CoV-2-Infizierter mit $16-20 \%$ angegeben wurde [35], sie Anfang Juni bei $31 \%$ lag [36] und jetzt bei 
jüngerem Durchschnittsalter der Infizierten und milderem Verlauf der Krankheit bei 40-45\% liegt [37]. Gandhi et al. [38] haben epidemiologische und klinische Befunde zusammengetragen, die dafür sprechen können, dass Masken (über den Schutz anderer (Fremdschutz) hinaus) das „inoculum“, d.h. die Dosis des Virus für den Maskenträger reduzieren (Eigenschutz) und zu milderen und asymptomatischen Verlaufsformen führen könnten. Die Hypothese, dass das Tragen von Masken in der Öffentlichkeit helfen könnte, den Schweregrad der Erkrankung zu reduzieren und dazu beitragen könnte, dass ein größerer Anteil an neuen Infektionen asymptomatisch verläuft [38, 39], bedarf dringend weiterer Untersuchungen. Keine Frage, die „Variolation“-Hypothese ist eine Hypothese, aber eine, die deutlich besser begründet ist als Ihre Sorge, Masken „müssen“ ... „im öffentlichen Raum sogar als ein Infektionsrisiko betrachtet werden " [1] (Seite 292) und ihre Schlussfolgerung, dass das Tragen von Masken im öffentlichen Raum „sogar potenziell kontra-produktiv“... „ist“ [1] (Seite 293). Beim derzeitigen Kenntnisstand halte ich vorsichtigere Formulierungen und eine ergebnisoffenere Einstellung für angebrachter. Es ist kein „Faktum, dass Masken in der Öffentlichkeit mehr Schaden als Nutzen bringen können“ [1] (Seite 292), im Gegenteil, es spricht vieles dafür, dass es eher umgekehrt ist.

Auch Ihre zurückhaltende Erörterung einer möglichen SARS-CoV-2-Übertragung durch Aerosole wird dem aktuellen Stand der wissenschaftlichen Diskussion nicht gerecht [40], reproduktionsfähige Viren ließen sich in der Raumluft von 2 Covid-19-Patienten in 2-4,8m Entfernung nachweisen und anzüchten [41].

\section{Public Health Aspekte: Rose's Präventionsparadox}

In einer Zeit, in der aktualisiertes Evidenz-basiertes Wissen als Goldstandard für ärztliches Handeln gilt [32], ist es hilfreich an das Dilemma jeder Prävention zu erinnern. Das Präventionsparadox, das der englische Epidemiologe G. Rose Anfang der 80er Jahre am Beispiel der koronaren Herzkrankheit und des Risikofak- tors Cholesterin ausführlich erörtert hat $[42,43]$, beinhaltet, dass eine Maßnahme, die für den betroffenen Einzelnen von großem Nutzen ist, der Gesamtbevölkerung oft wenig nützt und umgekehrt, dass eine Maßnahme, die für die Gesamtbevölkerung von großem Nutzen ist, dem Einzelnen oft wenig hilft. Rose hat aus diesem Paradox 2 unterschiedliche Präventionsstrategien abgeleitet: prophylaktische Maßnahmen für die Gesamtbevölkerung müssen für jedermann durchführbar und ohne Nebenwirkungen sein. Maßnahmen bei Individuen mit hohem Risiko rechtfertigen - nach individueller Risk-Benefit-Abwägung - den Einsatz von aggressiveren, mit Nebenwirkungen behafteten Maßnahmen.

Die beiden Präventionsstrategien, die Bevölkerungsstrategie und die Hoch-Risiko-Strategie, fokussieren auf unterschiedliche Behandlungsgruppen, auf „sick populations“ bzw. „sick patients“ [43]. Sie schließen einander nicht aus, sondern ergänzen sich. Im Falle der SARS-CoV-2-Pandemie beinhaltet die Bevölkerungsstrategie, die auf eine Verlangsamung der Ausbreitung des Virus in der Bevölkerung zielt, die für jedermann durchführbaren Maßnahmen wie Verzicht auf Massenveranstaltungen, Meiden sozialer Kontakte, Abstand halten und häufiges Händewaschen, bei potentiell Infizierten auch Quarantäne. Die Hoch-Risiko-Strategie zielt auf den Schutz älterer Menschen (Kontakte mit Großeltern meiden, Besuchsverbote in Krankenhäusern und Altenheimen), die Isolierung nachweislich Infizierter und die symptomadaptierte ambulante und stationäre Behandlung von Patienten mit hohem Risiko (ältere Menschen mit Begleitkrankheiten wie Herzerkrankungen, hohem Blutdruck, Diabetes mellitus oder chronischen Lungenerkrankungen), inklusive - wo nötig - stationärer Behandlung auf Intensivstation und ggf. maschineller Beatmung.

Ein wissenschaftstheoretisches Problem ist, dass - im Beispiel der koronaren Herzkrankheit, an dem Rose das Präventionsparadox erläutert hat [42] - viele Beobachtungsstudien und epidemiologische Studien die Effektivität einer Bevölkerungsstrategie (Aufgabe des Nikotin- konsums, diätetische Maßnahmen zur Senkung des Serumcholesterins, Vermeidung von Übergewicht und mehr körperliche Aktivität) zwar belegen [44], im Vergleich zu Meta-Analysen randomisierter Studien zur medikamentösen Senkung des Cholesterinspiegels mit 170 000 Personen [45] „underpowert“ und in der EBM-Pyramide [32] als geringwertiger erscheinen. Verschwiegen wird dabei, dass für eine Primärprävention mit Statinen die NNT (number need to treat) 1:500 beträgt, eine für die individuelle Beratung eines „sick patient“ völlig irrelevante Zahl [46]. Verschwiegen wird auch, dass viele der „Panelisten“ der großen Statin-Meta-Analyse ihre Interessenkonflikte und Verbandelungen mit der Industrie nicht offen gelegt haben [47] und durch solche Dinge - wie auch aus anderen Studien bekannt [48] - die Evidenz-basierte Medizin für andere Zwecke "gekapert“ und ihre Glaubwürdigkeit untergraben wird [48].

Als Ärzte, die wir individuelle Patienten behandeln und keine Populationen, sind wir meist blind für den Public-HealthBlick auf Populationen und den Gesamtblick auf die globale SARS-CoV-2-Pandemie. Wissenschaftler des Washingtoner Institute of Health Metrics and Evaluation (IHME) haben hochgerechnet, dass bei Fortsetzung der aktuellen Trends die Gesamtzahl der jetzt 1 Million betragenden Covid-19-Todesfälle [49] Anfang Januar 2021 weltweit 2, 5 Millionen betragen wird, eine Zahl, die möglicherweise auf 1,8 Millionen gesenkt werden könnte, wenn jedes Land eine Pflicht zum Tragen von Masken in der Öffentlichkeit einführen würde [50].

\section{Ethische Aspekte: das „precautionary principle“}

Anfang April haben Greenhalgh et al. [16] - ähnlich wie Sie Monate später in Ihrer o. a. Übersichtsarbeit [1] - die wissenschaftliche Literatur zu „face masks for the public" hinsichtlich Evidenz und interner und externer Validität gesichtet und erörtert. Obwohl Trisha Greenhalgh eine leidenschaftliche Kämpferin für eine Evidenz-basierte Medizin ist [51], 
berücksichtigen sie und ihre Mitautoren in ihrer Analyse nicht nur randomisierte kontrollierte Studien, sondern auch "good reasons“ und „substantial indirect evidence“ [16] wie experimentelle Befunde zu Aerosolen, hohes Übertragungsrisiko durch asymptomatisch Infizierte und „modelling studies“. Bei Einschluss auch solcher, in der EBM-Pyramide der Studien zur Evidenz [32] geringer gewichteter Befunde in die Gesamtbewertung (wie Observationsstudien zu hohen Infektionsraten (und erhöhter Mortalität) bei Beschäftigten im Gesundheitswesen [52, 53], hoher Infektionsrate bei Singen im Chor [54] u.a.m.) kommen sie jedoch zu erheblich anderen Interpretationen und Schlussfolgerungen als Sie.

Um das „precautionary principle“, „a strategy for approaching issues of potential harm when extensive scientific knowledge on the matter is lacking " [55, 56], an einem Alltagsbeispiel zu verdeutlichen: Brauche ich wirklich statistische Evidenz, um bei Nebel und regennasser Straße im Gebirge auf ein Überholmanöver zu verzichten, oder reicht dazu nicht „gesunder Menschenverstand'? Die ethische Frage, vor der ärztliche Gremien und politische Entscheidungsträger im April bei der Diskussion um Lockerungen des Lockdowns standen, ob mündige Bürger dazu ermutigt werden sollten, Masken zu ragen, haben Greenhalgh et al. [16] im April mit „we believe they should" beantwortet. Bezüglich des Nutzens präventiver Maßnahmen sei - allen Einschränkungen zum Trotz, die Sie gegen einen systematischen Review und Meta-Analyse von Observationsstudien (ohne Einbeziehung randomisierter Studien) vorbringen mögen - auf die Übersicht von Chu et al. [22] verwiesen.

Nach den sommerlichen Lockerungen der Maßnahmen zur Pandemieeindämmung in vielen Ländern stellt sich die Frage des Tragens von Masken in der Öffentlichkeit bei seit Wochen wieder z.T. rasant steigenden Infektionsraten in den USA und zahlreichen europäischen Ländern erneut - wenn auch in anderen Ländern mit stärkerer Dringlichkeit als hierzulande. Das einem präventiven Tragen von Masken zugrundeliegende ethische
Prinzip „The ethical justification for face coverings is their utility in preventing transmission of serious disease to community members" [57] gilt unverändert. Der altruistischen Begründung zum Tragen von Masken in der Öffentlichkeit stellt die o.a. enörterte Variolation-Hypothese einen möglichen individuellen und gesellschaftlichen Nutzen zur Seite.

\section{Handeln in Zeiten fehlenden expliziten Wissens}

Zur Gewinnung Evidenz-basierten Wissens zur Frage des Tragens von Masken in der Öffentlichkeit benötigen wir Zeit und Geduld-Zeit, in der sich die Pandemie weiter ausbreiten kann. „In the face of a pandemic the search for perfect evidence may be the enimy of good policy." und ..."it is time to act without waiting for randomised controlled trial evidence." (Greenhalgh et al. [16]).

Bleiben Sie gesund, egal ob Sie nur eine Maske im Krankenhaus in Bereichen höheren Infektionsrisikos zum Eigenschutz tragen oder - trotz fehlender Evidenz durch randomisierte Studien - auch eine Maske zum Eigenschutz und Fremdschutz in der Öffentlichkeit tragen!

Mit freundlichen Grüßen

Ottmar Leiß

Internist und Gastroenterologe

Mitglied der KRINKO 1998-2016

\section{Interessenkonflikt}

Der Autor gibt an, dass kein Interessenkonflikt besteht.

\section{Autorinnen/Autoren}

\section{Ottmar Leiß}

Internist und Gastroenterologe
Korrespondenzadresse

Prof. Dr. med. Ottmar Leiß Internist/Gastroenterologe Bodelschwinghstraße 14 65191 Wiesbaden

Deutschland

E-Mail: OLeiss@web.de

\section{Literatur}

[1] Kappstein I. Mund-Nasen-Schutz in der Öffentlichkeit: Keine Hinweise für eine Wirksamkeit. Krankenhaushygiene up2date 2020; 15: 279-297

[2] Kappstein I. Empfehlungen der „Richtlinie“ - Was mache ich anders? Krankenhaushygiene up2date 2009; 4: 9-294

[3] Kappstein I. Nosokomiale Infektionen. Prävention - Labordiagnostik - antimikrobielle Therapie. Stuttgart: Georg Thieme Verlag; 2009

[4] Leung NHL, Chu DKW, Shiu EYC et al. Respiratory virus shedding in exhaled breath and efficacy of face masks. Nature Med 2020; 26: 676-680

[5] World Health Organization. Non-pharmaceutical public health measures for mitigating the risk and impact of epidemic and pandemic influenza. 2019: Verfügbar unter (Zugriff 05.10.20): https://apps.who. int/iris/bitstream/handle/10665/329438/ 9789241516839-eng.pdf?ua $=1$

[6] World Health Organization. Advice on the use of masks in the context of COVID-19. Interim guidance. 2020: Verfügbar unter (Zugriff 05.10.20): https://apps.who.int/ iris/bitstream/handle/10665/331693/ WHO-2019-nCov-IPC_Masks-2020.3-eng. pdf? sequence $=1$ \&is Allowed $=y$

[7] World Health Organization. Advice on the use of masks in the context of COVID-19. Interim guidance. 05.06 2020: Verfügbar unter (Zugriff 05.10.20): https://apps. who.int/iris/bitstream/handle/10665/ 331693/WHO-2019-nCov-IPC_Masks2020.3-eng.pdf? sequence $=1$ \&isAllowed $=y$

[8] European Centre for Disease Prevention and Control (ECDC). Using face masks in the community. Reducing COVID-19 transmission from potentially asymptomatic or pre-symptomatic people through the use of face masks. 08.04 2020: Verfügbar unter (Zugriff 05.10.20): https://www. ecdc.europa.eu/sites/default/files/documents/COVID-19-use-face-masks-community.pdf

[9] Centers for Disease Control and Prevention (CDC). Use of Masks to Help Slow the Spread of COVID-19. 28.06 2020: https:// www.cdc.gov/coronavirus/2019-ncov/prevent-getting-sick/diy-cloth-face-coverings.html

[10] Robert Koch-Institut - RKI. Mund-NasenBedeckung im öffentlichen Raum als weitere Komponente zur Reduktion der Über- 
tragungen von COVID-19. Epid Bull 2020; 19: 3-5

[11] Guyatt GH, Oxman AD, Vist GE et al. Rating quality of evidence and strength of recommendations. GRADE: an emerging consensus on rating quality of evidence and strength of recommendations. BMJ 2008; 336: 924

[12] World Health Organization. Transmission of SARS-CoV-2: implications for infection prevention precautions. Scientific Brief. 2020: Verfügbar unter (Zugriff am 05.10.20): https://www.who.int/newsroom/commentaries/detail/transmissionof-sars-cov-2-implications-for-infectionprevention-precautions

[13] Bundesinstitut für Arzneimittel und Medizinprodukte. Hinweise des BfArM zur Verwendung von selbst hergestellten Masken (sog. „Community-Masken“), medizinischem Mund-Nasen-Schutz (MNS) sowie filtrierenden Halbmasken (FFP2 und FFP3) im Zusammenhang mit dem Coronavirus (SARS-CoV-2/Covid-19). Verfügbar unter (Zugriff am 05.10.20): https://www.bfarm. de/SharedDocs/Risikoinformationen/Medizinprodukte/DE/schutzmasken.html

[14] Goldmann E. Exaggerated risk of transmission of COVID-19 by fomites. Lancet 2020: doi:10.1016/S1473-3099(20)30561-2

[15] Ferguson NM, Laydon D, Nedjati-Gilani G et al. Impact of nonpharmaceutical interventions (NPIs) to reduce COVID-19 mortality and healthcare demand. 2020 : doi:10.25561/77482

[16] Greenhalgh T, Schmid MB, Czypionka T et al. Face masks for the public during the covid-19 crisis. BMJ 2020; 369: 1435 doi:10.1136/bmj.m1435

[17] Brooks JT, Butler JC, Redfield RR. Universal Masking to Prevent SARS-CoV-2 Transmission - The Time Is Now. JAMA 2020: doi:10.1001/jama.2020.13107

[18] Thagard P. How Scientist Explain Disease. Princeton, New Jersey: Princeton University Press; 1999

[19] Silverman WA. Where is the evidence? Debates in modern medicine New York: Oxford University Press; 1998

[20] Derry GN. What science is and how it works. Princeton, New Jersey: Princeton University Press; 1999

[21] Eikenberry SE, Mancuso M, Iboi E et al. To mask or not to mask: Modelling the potential for face mask use by the general public to curtail the COVID-19 pandemic. Infectious Disease Modelling 2020; 5: 293308

[22] Chu DK, Akl EA, Duda S et al. Physical distancing, face masks, and eye protection to prevent person-to-person transmission of SARS-CoV-2 and COVID-19: a systematic review and meta-analysis. Lancet 2020: doi:10.1016/S0140-6736(20)31142-9

[23] Prem K, Liu Y, Russell TW et al. The effect of control strategies to reduce social mixing on outcomes of the COVID-19 epidemic in Wuhan, China: a modelling study. Lancet
Public Health 2020: doi:10.1016/S24682667(20)30073-6

[24] Islam N, Sharp SJ, Chowell G et al. Physical distancing interventions and incidence of coronavirus disease 2019: natural experiment in 149 countries. BMJ 2020; 370: m2743 doi:10.1136/bmj.m2743

[25] Jones NR, Qureshi ZU, Temple RJ et al. Two metres or one: what is the evidence for physical distancing in covid-19? BMJ 2020; 370: m3223 doi:10.1136/bmj.m3223

[26] Lyu W, Wehby GL. Community use of face masks and COVID-19: Evidence from a natural experiment of state mandates in the US. Health Affairs 2020; 39: 1419-1425 doi:10.1377/hlthaff.2020.00818

[27] Kähler C], Hain R. Fundamental protective mechanisms of face masks against droplet infections. J Aerosol Sci 2020: 105617 doi:10.1016/j.jaerosci.2020.105617

[28] Leung NHL, Chu DKW, Shiu EYC et al. Respiratory virus shedding in exhaled breath and efficacy of facemasks. Nat Med 2020; 26: 676-680

[29] Bahl P, Bhattacharjee S, de Silva C et al. Face coverings and mask to minimise droplet dispersion and aerosolisation: a video case study. Thorax 2020; 75: 1024-1025 doi:10.1136/thoraxjnl-2020-215748

[30] Konda A, Prakash A, Moss GA et al. Aerosol filtration efficiency of common fabrics used in respiratory cloth masks. ACS Nano 2020; 14: doi:10.1021/acsnano.0c03252

[31] Morbidity and Mortality Weekly Report. Absence of apparent transmission of SARSCoV-2 from two stylists after exposure at a hair salon with a universal face covering policy - Springfield, Missouri. 2020: https://www.cdc.gov/mmwr/volumes/69/ wr/pdfs/mm6928e2-H.pdf (Zugriff 05.10.20)

[32] Howick J. The Philosophy of Evidence-Based Medicine. Wiley-Blackwell: BMJ Books; 2011

[33] Tao X, Garron T, Agrawal AS et al. Characterization and Demonstration of the value of a lethal mouse model of Middle East Respiratory Syndrome coronavirus infection and disease. J Virol 2016; 90: 57-67

[34] Memoli MJ, Czajkowski L, Reed S et al. Validation of the wild-type influenza $A$ human challenge model H1N1pdMIST: an A (H1N1)pdm09 dose-finding investigational new drug study. Clin Infect Dis 2015; 60: 693-702

[35] Buitrago-Garcia D, Egli-Gany D, Counotte $\mathrm{MJ}$ et al. The role of asymptomatic SARSCoV-2 infections: rapid living systematic review and meta-analysis. medRxiv 2020: doi:10.1101/2020.04.25.20079103

[36] Buitrago-Garcia D, Egli-Gany D, Counotte $\mathrm{MJ}$ et al. Occurrence and transmission potential of asymptomatic and presymptomatic SARS-CoV-2 infections: A living systematic review and meta-analysis. PLoS Med 2020: doi:10.1371/journal. pmed.1003346
[37] Oran DP, Topol EJ. Prevalence of Asymptomatic SARS-CoV-2 Infection: A Narrative Review. Ann Intern Med 2020: doi:10.7326/M20-3012

[38] Gandhi M, Beyrer C, Goosby E. Masks do more than protect others during COVID19: reducing the inoculum of SARS-CoV-2 to protect the wearer. J Gen Intern Med 2020: doi:10.1007/s11606-020-06067-8

[39] Gandhi M, Rutherford GW. Facial Masking for Covid-19 - Potential for "Variolation" as We Await a Vaccine. N Engl J Med 2020: doi:10.1056/NEJMp2026913

[40] Zhanga R, Lib Y, Zhang AL et al. Identifying airborne transmission as the dominant route for the spread of COVID-19. PNAS 2020; 117: 14857-14863

[41] Lednicky JA, Lauzardo M, Fan ZH et al. Viable SARS-CoV-2 in the air of a hospital room with COVID-19 patients. medRxi 2020: doi:10.1101/2020.08.03.20167395

[42] Rose G. Strategy of prevention: lessons from cardiovascular disease. BMJ 1981; 282: 1847-1851

[43] Rose G. Sick individuals and sick populations. Int J Epidemiol 1985; 14: 32-38

[44] Eckel RH, Jakicic JM, Ard JD et al. ACC/AHA Guideline on Lifestyle Management to Reduce Cardiovascular Risk. A Report of the American College of Cardiology/American Heart Association Task Force on Practice Guidelines. Circulation 2014; 129: S49S73

[45] Cholesterol Treatment Trialists (CTT) Collaboration. Efficacy and safety of more intensive lowering of LDL cholesterol: a meta-analysis of data from 170000 participants in 26 randomised trials. Lancet 2010; 376: 1670-1681

[46] Hartzband P, Gropman J. Where am I in the numbers? Hartzband P, Gropman J. Your Medical Mind. How to Decide What Is Right for You. London: Penguin Books; 2011: 929

[47] Lenzer J. Majority of panellist on controversial new cholesterol guideline have current or recent ties to drug manufactures. BMJ 2013; 347: f6989

[48] Jureidini ], McHenry LB. The Illusion of Evidence-Based Medicine. Exposing the crisis of credibility in clinical research. Adelaide, South Australia: Wakefield Press; 2020

[49] Mega ER. COVID has killed more than one million people. How many more will die? Nature 2020: doi:10.1038/d41586-02002762-y

[50] Institute of Health Metrics and Evaluation (IHME). COVID-10 Projections. Verfügbar unter (Zugriff 06.10.20): https://covid19. healthdata.org/global?view=daily-deaths\&tab=trend

[51] Greenhalgh T, Howick J, Maskrey N et al. Evidence based medicine: a movement in crisis? BMJ 2014; 348: g3725 
[52] Allison TA, Oh A, Harrison KL. Extreme Vulnerability of Home CareWorkers During the COVID-19 Pandemic - A Call to Action. JAMA Intern Med 2020: doi:10.1001/jamainternmed.2020.3937

[53] Janssens U, Schlitt A, Hein A et al. SARSCoV-2 Infektion bei Mitarbeiterinnen und Mitarbeitern im Gesundheitswesen - Bedeutung der RT-PCR Testung. AWMF online 2020: doi:10.1111/pai.13265

[54] Read R. A choir decided to go ahead with rehearsal. Now dozens of members have COVID-19 and two are dead. Los Angeles Times. 29.03 2020: Verfügbar unter (Zugriff 06.10.20): https://www.msn.com/enus/news/us/a-choir-decided-to-go-aheadwith-rehearsal-now-dozens-of-membershave-covid-19-and-two-are-dead/arBB11T9wB
[55] European Commission. The precautionary principle: decision-making under uncertainty. 2017: Verfügbar unter (Zugriff 06.10.20): https://ec.europa.eu/environment/integration/research/newsalert/pdf/ precautionary_principle_decision_making_under_uncertainty_FB18_en.pdf

[56] Reis J, Spencer PS. Decision making under uncertainty in environmental health policy: new approches. Environ Health Prev Med 2019; 24: 57

[57] Gostin LO, Cohen IG, Koplan JP. Universal masking in the United States - The role of mandates, health education, and the CDC. JAMA 2020: doi:10.1001/jama.2020.15271

\section{Publikationshinweis}

Leserbriefe stellen die Meinung des Autors dar und entsprechen nicht unbedingt der Meinung von Herausgebern oder Verlag. Herausgeber und Verlag behalten sich vor, Leserbriefe nicht, gekürzt oder in Auszügen zu veröffentlichen.

Bibliografie

Krankenhaushygiene up2date 2020; 15: 327-332

DOI https://doi.org/10.1055/a-1289-8435

ISSN 1862-5797

online publiziert 27.10 .2020

(c) 2020. Thieme. All rights reserved. Georg Thieme Verlag KG, Rüdigerstraße 14, 70469 Stuttgart, Germany 